\title{
Comparison of CSF neurofilament light chain, neurogranin, and tau to MRI markers
}

Michelle M. Mielke ${ }^{a, b *}$, Scott Przybelski ${ }^{a}$, Tim Lesnick ${ }^{a}$, Silke Kerne, Henrik Zetterberg, e,f,g, haj Klennow ${ }^{e, f}$, David S. Knopman ${ }^{b}$, Jonathan Graff-Radford ${ }^{b}$, Ronald C. Petersen ${ }^{\mathrm{a}, \mathrm{b}}$, Clifford R. Jack Jr ${ }^{\mathrm{c}}$, Prashanthi Vemuric

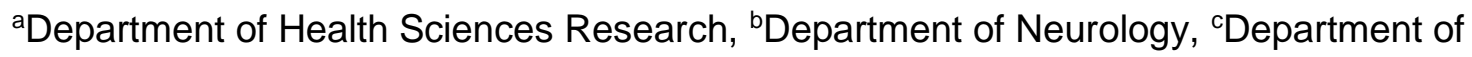
Radiology, ${ }^{d}$ Department of Psychiatry and Psychology, Mayo Clinic, Rochester, MN 55905, USA ${ }^{e}$ Department of Psychiatry and Neurochemistry, Institute of Neuroscience and Physiology, The Sahlgrenska Academy at the University of Gothenburg, Mölndal, Sweden

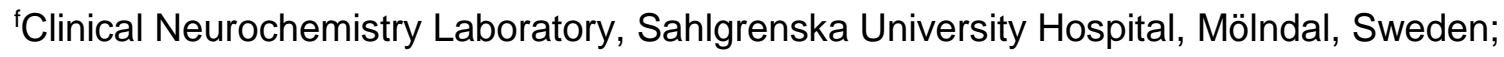
9Department of Neurodegenerative Disease, UCL Institute of Neurology, London, UK; and hUK Dementia Research Institute at UCL, London, UK

${ }^{*}$ Corresponding Author: Michelle M. Mielke, PhD, Department of Health Sciences Research, Mayo Clinic, 200 First Street SW, Rochester, MN 55905, USA

Email: Mielke.Michelle@mayo.edu, Telephone: 507-293-1069; Fax: 507-284-1516. 


\begin{abstract}
Introduction: We determined whether cerebrospinal fluid (CSF) neurofilament light (NfL), neurogranin $(\mathrm{Ng})$, and total-tau (t-tau) differentially mapped to magnetic resonance imaging (MRI) measures of cortical thickness, microstructural integrity (corpus callosum and cingulum fractional anisotropy [FA]), and white matter hyperintensities (WMH).

Methods: Analyses included 536 non-demented Mayo Clinic Study of Aging participants with CSF NfL, $\mathrm{Ng}$, t-tau, amyloid beta $(A \beta) 42$ and longitudinal MRI scans. Linear mixed models assessed longitudinal associations between CSF markers and MRI changes.

Results: Higher CSF NfL was associated with decreasing microstructural integrity and WMH. Higher t-tau was associated with decreasing temporal lobe and Alzheimer's disease $(A D)$ meta region of interest (ROI) cortical thickness. There was no association between $\mathrm{Ng}$ and any MRI measure. CSF A 42 interacted with $\mathrm{Ng}$ for declines in temporal lobe and AD meta ROI cortical thickness and cingulum FA.

Discussion: CSF NfL predicts changes in white matter integrity, t-tau reflects nonspecific changes in cortical thickness, and $\mathrm{Ng}$ reflects $\mathrm{AD}$-specific synaptic and neuronal degeneration.

Keywords: Alzheimer's; amyloid; cerebrospinal fluid; cohort study; cortical thickness; magnetic resonance imaging; neurodegeneration; neurofilament light chain; neurogranin; total tau; white matter integrity.
\end{abstract}




\section{Introduction}

Neurodegeneration is the pathological process most strongly associated with clinical symptoms, as compared to proteins [e.g., amyloid-beta, tau], in Alzheimer's disease (AD).\{Terry RD, et al. Ann Neurol 1991; 30:572-580; Giannakopoulos P, et al.

Neurology 2003;60:1495-1500\} There are many potential fluid and imaging neurodegeneration markers that may be specific or non-specific to $A D$ and other pathologies across brain regions. It is important to understand what information different biomarkers provide to aid in both diagnosis and prognosis, especially because each marker is likely to be reflective of a different composite of pathological processes.

Under the new AT(N) scheme of the NIA-AA Research Framework \{Jack CR Jr, et al. Alzheimers Dement 2018;14:535-62\}, proposed cerebrospinal fluid (CSF) markers of neurodegeneration $(\mathrm{N})$ now include total-tau ( $\mathrm{T}$-tau), neurofilament light chain $(\mathrm{NfL})$, and neurogranin $(\mathrm{Ng})$. Several studies have examined and compared these three CSF neurodegeneration markers for improving clinical diagnosis and for predicting cognitive decline \{e.g., Mattsson N, et al. EMBO Molecular Medicine 2016;8:1184-1196; Tarawneh R, et al. JAMA Neurol 2016;73(5):561-571; Mattsson N, et al. JAMA Neurol 2017;74(5):557-566; Kern S, et al., JAMA Neurol 2019;76:187-93; Bos I, et al. Alzheimer's \& Dementia 2019;15:644-654; Merluzzi AP, et al. Alzheimer's \& Dementia: Translational Research \& Clinical Interventions 2019;5:129-138\}. However, few studies directly examined how CSF $\mathrm{T}$-tau, $\mathrm{NfL}$, and $\mathrm{Ng}$ related to longitudinal changes in multiple neuroimaging markers of neurodegeneration, especially in a community based population. Some studies have suggested that the effects of elevated CSF T-tau and 
$\mathrm{Ng}$ for hippocampal atrophy is amyloid-dependent whereas CSF NfL is associated with hippocampal atrophy regardless of amyloid status \{Mattsson N, et al. EMBO Molecular Medicine 2016;8:1184-1196; Portelius E, et al. Brain 138:3373-3385\}. However, another study did not find the effects of CSF T-tau on cerebral atrophy to be amyloid-dependent \{Pereira JB, et al. Neurobiology of Aging 2017; 14-29\}. CSF NfL has been consistently shown to be associated with white matter integrity, as assessed by diffusion tensor imaging (DTI), and with white matter hyperintensities (WMH) \{e.g., Moore EE, et al.

\section{8; Neurobiology of Aging 70:233-241; Kim WH, et al. Neurolmage: Clinical}

2019;21:101586\}, independent of amyloid pathology. However, the association between

CSF Ng or T-tau with white matter are less well known. The goal of the present study was to determine whether CSF T-tau, NfL, and $\mathrm{Ng}$ differentially map to specific MRI measures of neurodegeneration and white matter, and whether these associations differed by elevated brain amyloid in a large community based non-demented population.

\section{Methods}

Participants

The Mayo Clinic Study on Aging (MCSA) is a prospective population-based study examining the epidemiology of cognitive decline and risk of $\mathrm{MCl}$ among residents living in Olmsted County, Minnesota \{Roberts RO, et al. Neuroepidemiology 2008\}. In 2004, Olmsted County residents between the ages of 70 and 89 were enumerated using the Rochester Epidemiology Project medical records-linkage system in an age- and sexstratified random sampling design \{St. Sauver JL, et al. Int J Epidemiol 2012;41:16141624\}. The study was extended to include those aged 50 and older in 2012. MCSA 
visits include an interview by a study coordinator, physician examination, and neuropsychological testing \{Roberts RO, et al. Neuroepidemiology 2008\}. Clinical follow-up visits occur at 15 month intervals. Participant demographics (age, sex, and years of education) and medical history were ascertained at the in-clinic examination. At each visit, participants are asked if they would be willing to undergo a lumbar puncture for the collection of CSF or to have neuroimaging. The current analyses included 536 non-demented MCSA participants who had CSF assays of NfL, $\mathrm{Ng}$, T-tau and A $\beta 42$, and MRI measures of cortical thickness or white matter (corpus callosum FA, cingulum FA, and/or white matter hyperintensity volume). We do not have all MRI measures available in all 536 individuals because the $2.7 \mathrm{~mm}$ voxel size DTI data used in this study started in 2009 and the WMH assessments were available in a subset of individuals. The study was approved by Mayo Clinic and Olmsted Medical Center Institutional Review Boards. Written informed consent was obtained from all participants.

Mild Cognitive Impairment (MCl) and dementia diagnostic determination

Clinical diagnoses were determined by a consensus committee of those who evaluated each participant. Cognitive performance was compared with the age-adjusted scores of cognitively unimpaired (CU) individuals previously obtained using Mayo's Older American Normative Studies \{lvnik RJ, et al. Clin Neuropsychol 1992;83-104\}. Participants with scores around 1.0 SD below the age-specific mean in the general population were considered for possible cognitive impairment. The operational definition of $\mathrm{MCl}$ was based on clinical judgment including a history from the patient and 
informant. Published criteria were used for the diagnosis: cognitive complaint, cognitive function not normal for age, essentially normal functional activities, no dementia \{Petersen RC, J Intern Med 2004;256:183-194\}. A final decision was made after considering education, occupation, visual or hearing deficits, and reviewing all other participant information. The diagnosis of dementia was based on published criteria \{American Psychiatric Association. Diagnostic and Statistical Manual of Mental Disorders (DSM-IV). 4th ed. Washington, D.C.: American Psychiatric Association; 1994\}. Participants who performed in the normal range and did not meet criteria for $\mathrm{MCl}$ or dementia were deemed CU. The consensus committee was blinded to CSF and neuroimaging results when determining the clinical diagnosis.

\section{Lumbar punctures and CSF measurements}

Fasting lumbar punctures were performed early in the morning in the lateral decubitus position from the L3 and L4 intravertebral space using a 20 or 22 gauge Quincke needle. Two cc of CSF were used to evaluate routine markers (glucose, protein, cell count). The remainder was divided into $0.5 \mathrm{cc}$ aliquots and stored at $-80^{\circ} \mathrm{C}$. Samples had not undergone a freeze-thaw cycle prior to being pulled. As previously described \{Mielke MM, et al. Alzheimers Dement 2019;15(11):1437-1447\}, participants who underwent a lumbar puncture, compared to those who did not, were younger, more likely to be male, and generally healthier. However, clinical diagnosis (e.g., $\mathrm{CU}, \mathrm{MCl}$, dementia) was not associated with participation in a lumbar puncture.

CSF AB42 and T-tau were measured with automated electrochemiluminescence Elecsys immunoassays (Roche Diagnostics), as previously described \{Mielke MM, et al. 
Alzheimers Dement 2019;15(11):1437-1447\}. A CSF Aß42 level<1026 pg/mL was considered amyloid positive $(\mathrm{A}+)$ \{REF!!!\} We did not use the $\mathrm{P}$-tau/Aß42 ratio because we have previously shown a high correlation (Spearman rho $=0.98, p<0.001$ ) between CSF P-tau and CSF T-tau in this cohort. CSF NfL and Ng were measured using inhouse ELISAs; their assay characteristics and methods have been described in detail \{Kern S, et al. JAMA Neurol 2019;76:187-193; van Harten AC, Gaetani L, et al.

Alzheimers Res Ther 2018;10:8; Kvartsberg H, et al. Acta Neuropathol 2019;137:89$102\}$.

\section{Structural MRI}

Neuroimaging occurs at 15- or 30-month intervals. Structural magnetic resonance imaging (MRI) was acquired using standardized Magnetization Prepared - Rapid Gradient Echo (MPRAGE) sequences on 3T GE scanners (GE Medical Systems, Milwaukee, WI). A global cortical thickness AD measure was computed using a FreeSurfer (version 5.3)-derived temporal lobe cortical thickness composite of

entorhinal, fusiform, inferior temporal, and middle temporal ROIs ${ }^{20}$ from 3T MPRAGE scans.

DTI sequences were processed and analyzed for fractional anisotropy (FA) of the corpus callosum and of the cingulum \{Ramanan VK, et al. J Alzheimers Dis 2018;65:1345-1352; Vemuri P, et al. Ann Neurol 2018;84(5):705-716\}. Loss of white matter microstructural integrity measured using DTI has been shown to be a good indicator of axonal injury. We used the JHU atlas to regionally measure FA from DTI scans \{Oishi K, et al. Neuroimage 2009;46:486-499\}. We considered corpus callosum FA (combination of the genu, body, splenium from JHU atlas) because these 
interhemispheric connections are widely disrupted in aging and dementia \{Frederiksen KS, Dan Med J 2013;60(10):B4721\}. We considered cingulum FA (combination of the cingulum in the cingulate gyrus and cingulum adjoining the hippocampus from JHU atlas) because these tracts are specifically impacted in $A D$ \{Zhang $Y$, et al. Neurology $2007 ; 68(1): 13-19\}$

White matter hyperintensities on standard 2-dimensional Fluid-attenuated inversion recovery (FLAIR) imaging were segmented and edited by a trained imaging analyst using a semi-automated method \{Graff-Radford J, et. al. Brain 2019;142(8):2483-2491\}. WMH volume is presented as the percentage of total intracranial volume (TIV).

\section{Statistics}

Linear mixed models were used to assess the associations between each baseline CSF $\mathrm{N}$ marker and longitudinal changes in each MRI measure adjusting for age, sex, and CSF amyloid. Interactions between each CSF N marker and low CSF amyloid-beta 42 $(\mathrm{A}+)$ for change in MRI measures were assessed. Sensitivity analyses were conducted that included only those individuals who had all neuroimaging measures. Because we had multiple neuroimaging outcomes, we considered $p<0.01$ as statistically significant.

\section{Results}

\section{Participant characteristics}

The characteristics of the 536 participants with CSF and any MRI neuroimaging

measure are shown in Table 1. The mean (standard deviation [SD]) age was 74.9 (7.3) years and education was $14.4(2.8)$ years. There were 304 men (57\%), $154(29 \%)$ with 
an APOE E4 allele, and 70 (13\%) whom had a clinical consensus diagnosis of $\mathrm{MCl}$. The participants had an average of 2.4 (1.8) follow-up years and $2.2(1.0)$ visits. Of the 536 participants, 533 had concurrent CSF and cortical thickness, 273 concurrent CSF and DTI, and 211 concurrent CSF and WMH fraction; 204 (38.1\%) had all neuroimaging outcomes. Compared to those with all imaging parameters, those without were significantly older, more frequently male, had higher levels of all CSF measures, and had a greater number of years of follow-up (Table 1).

Associations between each CSF ' $N$ ' marker and both cross-sectional and longitudinal neuroimaging outcomes

In multivariate analyses adjusting for age, sex, and CSF A $\beta 42$, higher CSF $\mathrm{Ng}$ was cross-sectionally associated with higher corpus callosum $\mathrm{FA}(\mathrm{b}(\mathrm{se})=0.186(0.070)$, $\mathrm{p}=0.008$ ). In addition, higher CSF NfL was associated with lower cortical thickness in the frontal $(b(s e)=-0.229(0.045), p<0.001)$, parietal $(b(s e)=-0.167(0.043), p<0.001)$, and temporal $(\mathrm{b}(\mathrm{se})=-0.144(0.043), \mathrm{p}<0.001)$ lobes and in the $A D$ signature region $(b(s e)=-0.162(0.045), p<0.001)$. There were no cross-sectional associations between CSF T-tau and any of the markers (Table 2).

Longitudinally, in multivariate analyses, higher CSF T-tau was associated with declines in temporal lobe cortical thickness $(b(s e)=-0.189(0.068), p=0.006)$ and the $A D$ signature region $(b(s e)=-0.281(0.073), p<0.001$; Table 2 and Figure 1). Higher CSF $\mathrm{NfL}$ was associated with declines in the Corpus Callosum $(b(s e)=-0.283(0.096)$, 
$\mathrm{p}=0.003)$ and Cingulum $(\mathrm{b}(\mathrm{se})=-0.308(0.096), \mathrm{p}=0.006) \mathrm{FA}$. There were no associations between CSF $\mathrm{Ng}$ and change in any neuroimaging measures.

Interactions between each CSF ' $N$ ' marker and CSF A 342 for both cross-sectional and longitudinal neuroimaging outcomes

Cross-sectionally, neither CSF NfL, nor $\mathrm{Ng}$ interacted with CSF Aß42 in relation to any neuroimaging outcome in multivariate models. However, higher CSF T-tau was associated with lower temporal lobe thickness among participants who were amyloid positive compared to amyloid negative $(b(s e)=-0.222(0.84), p=0.008$; Table 3$)$.

Longitudinally, the association between CSF T-tau or NfL and change in any of the neuroimaging markers did not differ by amyloid status. However, higher CSF $\mathrm{Ng}$ was associated with greater declines in temporal lobe thickness $(b(s e)=-0.464(0.140)$, $p<0.001), A D$ signature region thickness $(-0.471(0.151), p=0.002)$, and cingulum FA ($0.662(0.240), p=0.006)$ in amyloid positive individuals compared to amyloid negative individuals (Table 3; Figure 2).

\section{Sensitivity Analyses}

In sensitivity analyses, we restricted the sample size to those whom had all imaging modalities and reassessed the associations between each CSF ' $N$ ' marker and both cross-sectional and longitudinal neuroimaging outcomes (Supplementary Table 1). The results were essentially the same. Similarly, we also reanalyzed interactions between each CSF ' $\mathrm{N}$ ' marker and CSF A 42 for the neuroimaging outcomes and the results 
were compatible (Supplementary Table 2). In addition, we did not identify interactions between each CSF marker and sex for any of the outcomes.

\section{Discussion}

There are multiple markers of neurodegeneration in the CSF and via neuroimaging, some of which may be more specific to AD. In the present study, we examined whether CSF T-tau, NfL, and $\mathrm{Ng}$ differentially associated with specific MRI measures of neurodegeneration and white matter, and whether these associations differed by elevated brain amyloid in a community based non-demented population. Our results suggest that CSF NfL, $\mathrm{Ng}$, and T-tau differentially correspond to MRI measures of neurodegeneration and white matter. CSF Ng was most specific for AD-associated neurodegeneration and reflected greater declines in AD-related imaging biomarkers especially in the presence of amyloidosis. In contrast, the associations between CSF NfL and neuroimaging measures did not differ by CSF amyloid and were strongly associated with white matter indices. Similarly, CSF T-tau reflected non-specific neurodegenerative changes in both cortical thickness and white matter and did not differ by CSF amyloid.

Neurogranin is a post-synaptic protein that is specific to neurons, important for synaptic plasticity, and abundant in excitatory neurons in the cortex and hippocampus \{Represa A, et al. J Neurosci 1990;10(12):3782-3792\}. Previous studies of clinic-based samples have suggested that $\mathrm{Ng}$ is specific to AD by demonstrating that CSF $\mathrm{Ng}$ is only elevated in $A D$ patients compared to controls, but not those with frontotemporal dementia, Lewy 
body dementia, Parkinson's disease or other neurodegenerative diseases $\{$ Wellington H, et al., 2016 Neurology 86(9):829-35; Portelius E, et al., Acta Neuropathol 2018;136(3):363-376\}. Cross-sectionally, studies have shown that the association between hippocampal atrophy and CSF $\mathrm{Ng}$ is amyloid-dependent \{Mattsson $\mathrm{N}$, et al. EMBO Molecular Medicine 2016;8:1184-1196; Pereira JB, et al. Neurobiol Aging 2017;58:14-29\}. Our current results further support an amyloid-dependent prognostic effect of elevated CSF $\mathrm{Ng}$ for declines in temporal and AD signature region cortical thickness and cingulum FA. The association between CSF Ng and white matter enhances previous research suggesting an association between CSF $\mathrm{Ng}$ and white matter integrity $\{$ Kim WH, et al., Neurolmage: Clinic 2019;21:101586\}. Notably, in our results CSF Ng was more strongly associated with Cingulum FA than corpus callosum in Table 3 among A+ participants. Disruption of the limbic white matter pathways is well known in $A D$, supporting these findings of the cingulum $F A$ in the presence of amyloidosis \{Bozoki AC, et al. Hum Brain Mapp 2012;33(8):1792-1802; Zhang Y, et al. Neurology 2007;68(1):13-19\}.

Studies examining the relationship between CSF T-tau and neuroimaging markers of neurodegeneration, and whether the associations are AD-specific, are less clear. For example, one study reported that high CSF T-tau was associated with lower hippocampal volume and FDG-PET hypometabolism only among those who were amyloid positive \{Mattsson N, et al. EMBO Mol Med (2016);8:1184-1196\}. However, another study did not find an amyloid-dependent effect of CSF T-tau on voxel-based cortical atrophy \{Pereira JB, et al. Neurobiology of Aging 2017; 14-29\}. Our results are 
somewhat related to these previous findings that used ADNI data. Cross-sectionally, there was an amyloid-dependent effect of CSF T-tau on temporal lobe cortical thickness and a trend for $A D$ signature region thickness. Longitudinally, there were no significant associations at the $p<0.01$ level but there were trends of associations for CSF T-tau with temporal lobe cortical thickness and for cingulum FA. Thus, while CSF T-tau has some specificity for AD-associated neurodegeneration, it does not appear to be as specific as CSF Ng. The non-specificity of CSF T-tau was also reflected by the associations with corpus callosum FA which is a more general (non-specific) marker of cognitive dysfunction.

CSF NfL has been consistently shown to be associated with white matter integrity, as assessed by DTI, and with white matter hyperintensities \{e.g., Moore EE, et al. 2018; Neurobiology of Aging 70:233-241; Kim WH, et al. Neurolmage: Clinical

$2019 ; 21: 101586\}$, independent of amyloid pathology. The present findings are in line with this past research both cross-sectionally and longitudinally. Surprisingly we did not find any associations of CSF NfL with WMH. Even though WMH are a result of continued WM degeneration over time, fractional anisotropy changes on DTI are seen prior to $\mathrm{WMH}$ damage and may likely reflect early damage immediately subsequent to the CSF NfL changes \{Maillard P, et al. AJNR Am J neuroradiol 2013;34(1):54-61\}.

A strength of the study is the community-based sample. Many previous studies that utilized clinic-based samples or ADNI likely have less pathology and are not representative of the general community. Another strength is that we evaluated all three 
neurodegeneration CSF biomarkers using longitudinal imaging outcomes. There are some limitations as well. We considered a limited number of imaging outcomes by using specific global measures. Further, we had limited numbers with all three sets of imaging outcomes measured longitudinally.

\section{Conclusion}

The three candidate CSF markers of neurodegeneration $(\mathrm{N})$ - NfL, $\mathrm{Ng}$, and T-tau may reflect different underlying pathophysiologies. 


\section{Acknowledgments}

Funding: This study was supported by funding from the National institutes of Health/National Institute on Aging grants U01 AG006786, R01 AG011378, R01 AG041851, R01 AG049704, and R01 AG034676. Additional funding came from the GHR Foundation, the Swedish Research Council (2015-02830), the European Research Council (681712), the Knut and Alice

Wallenberg Foundation, Frimurarestiftelsen, the Olav Thon Foundation, Swedish State Support for Clinical Research (ALF Västra Götalandsregionen), the Swedish state under the agreement between the Swedish Government and the county councils, the ALF-agreement (ALFGBG813921, ALFGBG-65930, ALF-GBG-716681,ALF GBG-771071), the Torsten Söderberg Foundation, The Swedish Alzheimer Foundation, Hjärnfonden Sweden, Stiftelsen Demensfonden, Stiftelsen Hjalmar Svenssons Forskningsfond, Stiftelsen Wilhelm och Martina Lundgrens Vetenskapsfond. HZ is a Wallenberg Scholar.

Conflict of Interest Disclosures: Dr. Mielke served as a consultant to Brain Protection Company and receives research support from the National Institutes of Health (R01 AG49704, P50 AG44170, U01 AG06786, RF1 AG55151), and unrestricted research grants from Biogen. Mr. Syrjanen has nothing to disclose. Mr. Przybelski and Mr. Lesnick have nothing to disclose. Dr. Kern has nothing to disclose. Dr. Zetterberg has served at advisory boards for Denali, CogRx, Roche Diagnostics, Samumed and Wave, has given lectures in symposia sponsored by Fujirebio, Alzecure and Biogen, and is a co-founder of Brain Biomarker Solutions in Gothenburg $A B$, a GU Ventures-based platform company at the University of Gothenburg. Dr. Blennow has served as a consultant or at advisory boards for Alzheon, BioArctic, Biogen, Eli Lilly, Fujirubio Europe, IBL International, Merck, Novartis, Pfizer, and Roche Diagnostics, and is a co-founder of Brain Biomarker Solutions in Gothenburg AB, a GU Ventures-based platform company at the University of Gothenburg. Dr. Knopman serves on a Data Safety Monitoring Board for Lundbeck Pharmaceuticals and for the DIAN study; is an investigator in clinical trials sponsored by Biogen, 
Lilly Pharmaceuticals, and the Alzheimer's Disease Cooperative Study; and receives research support from the National Institutes of Health. Dr. Graff-Radford has nothing to disclose. Dr. Petersen is a consultant for Roche, Inc., Merck, Inc., Biogen, Inc., Genentech, Inc., and GE Healthcare. He receives research support from the National Institutes of Health.Dr. Jack has provided consulting services for Eli Lilly. He receives research funding from the National Institutes of Health, and the Alexander Family Alzheimer's Disease Research Professorship of the Mayo Clinic. Dr. Vemuri has nothing to disclose. 
Table 1. Baseline characteristics for all participants and by status of complete data for all neuroimaging modalities

\begin{tabular}{|c|c|c|c|c|}
\hline Characteristic & $\begin{array}{c}\text { All }(\mathrm{N}=536) \\
N(\%) / \operatorname{mean}(S D)\end{array}$ & $\begin{array}{c}\text { Complete } \\
(\mathrm{N}=204) \\
N(\%) / \text { mean }(S D)\end{array}$ & $\begin{array}{c}\text { Not Complete }(\mathrm{n}=332) \\
N(\%) / \text { mean }(S D)\end{array}$ & $P$-value \\
\hline Age, years & $74.9(7.3)$ & $68.5(5.8)$ & $78.8(5.1)$ & $<0.001$ \\
\hline Male & $304(56.7 \%)$ & $96(47.1 \%)$ & $208(62.7)$ & $<0.001$ \\
\hline Education, years & $14.4(2.8)$ & $14.8(2.6)$ & $14.2(2.9)$ & 0.03 \\
\hline Presence of an $A P O E \varepsilon 4$ allele & $154(28.7 \%)$ & $69(33.8 \%)$ & $85(25.6 \%)$ & 0.04 \\
\hline Diagnosis of $\mathrm{MCl}$ & $70(13.1 \%)$ & $21(10.3 \%)$ & $49(14.8 \%)$ & 0.14 \\
\hline CSF A $1-42, \mathrm{pg} / \mathrm{ml}$ & $1188.8(591.4)$ & $1066.4(481.8)$ & $1264.1(638.8)$ & $<0.001^{*}$ \\
\hline CSF Total tau, $\mathrm{pg} / \mathrm{ml}$ & $248.9(102.9)$ & $211.7(81.3)$ & $271.7(108.1)$ & $<0.001^{*}$ \\
\hline CSF Neurogranin, $\mathrm{pg} / \mathrm{ml}$ & $185.7(70.2)$ & $172.2(64.2)$ & $194.0(72.6)$ & $<0.001^{*}$ \\
\hline CSF Neurofilament light chain, $\mathrm{pg} / \mathrm{ml}$ & $787.0(951.7)$ & $658.4(1170.4)$ & $866.0(779.4)$ & $<0.001^{*}$ \\
\hline Follow-up, years & $2.4(1.8)$ & $2.1(1.7)$ & $2.6(1.8)$ & 0.001 \\
\hline Number of visits & $2.2(1.0)$ & $1.9(0.8)$ & $2.4(1.0)$ & $<0.001$ \\
\hline
\end{tabular}

${ }^{*}$ Variable was log transformed in t-test. 
Table 2. Multivariable Associations between Each CSF 'N' Marker and both crosssectional and Longitudinal Neuroimaging Outcomes

\begin{tabular}{|c|c|c|c|c|}
\hline \multirow[b]{2}{*}{ Neuroimaging measure(z-score) } & \multicolumn{2}{|c|}{ CSF Total tau } & \multicolumn{2}{|c|}{ CSF Total tau*Time } \\
\hline & $b(s e)$ & $p$-value & $b(s e)$ & $\begin{array}{c}p- \\
\text { value }\end{array}$ \\
\hline \multicolumn{5}{|l|}{ Cortical Thickness } \\
\hline Frontal & $-0.074(0.045)$ & 0.103 & $-0.093(0.077)$ & 0.225 \\
\hline Parietal & $-0.072(0.043)$ & 0.091 & $-0.034(0.073)$ & 0.642 \\
\hline Occipital & $0.008(0.042)$ & 0.840 & $0.013(0.070)$ & 0.852 \\
\hline Temporal & $0.024(0.043)$ & 0.579 & $-0.189(0.068)$ & 0.006 \\
\hline$A D$ signature region & $-0.040(0.044)$ & 0.366 & $-0.281(0.073)$ & $<0.001$ \\
\hline \multicolumn{5}{|l|}{ White Matter Indices } \\
\hline Corpus Callosum FA & $0.191(0.075)$ & 0.012 & $-0.150(0.099)$ & 0.132 \\
\hline Cingulum FA & $-0.151(0.069)$ & 0.031 & $-0.135(0.116)$ & 0.245 \\
\hline \multirow[t]{2}{*}{ WMH Fraction } & $-0.025(0.071)$ & 0.725 & $0.087(0.074)$ & 0.240 \\
\hline & \multicolumn{2}{|c|}{ CSF Neurogranin } & \multicolumn{2}{|c|}{ CSF Neurogranin*Time } \\
\hline Neuroimaging measure(z-score) & $b(s e)$ & $\begin{array}{c}p- \\
\text { value }\end{array}$ & $b(s e)$ & $\begin{array}{c}p- \\
\text { value }\end{array}$ \\
\hline \multicolumn{5}{|l|}{ Cortical Thickness } \\
\hline Frontal & $-0.088(0.043)$ & 0.042 & $0.004(0.078)$ & 0.960 \\
\hline Parietal & $-0.074(0.041)$ & 0.068 & $0.016(0.074)$ & 0.832 \\
\hline Occipital & $-0.013(0.040)$ & 0.737 & $-0.037(0.071)$ & 0.608 \\
\hline Temporal & $0.008(0.041)$ & 0.844 & $-0.095(0.070)$ & 0.176 \\
\hline$A D$ signature region & $-0.038(0.042)$ & 0.371 & $-0.164(0.075)$ & 0.029 \\
\hline \multicolumn{5}{|l|}{ White Matter Indices } \\
\hline Corpus Callosum FA & $0.186(0.070)$ & 0.008 & $-0.206(0.102)$ & 0.043 \\
\hline Cingulum FA & $-0.079(0.065)$ & 0.225 & $-0.139(0.119)$ & 0.245 \\
\hline \multirow[t]{2}{*}{ WMH Fraction } & $-0.117(0.065)$ & 0.076 & $-0.003(0.077)$ & 0.964 \\
\hline & \multicolumn{2}{|c|}{ CSF NfL } & \multicolumn{2}{|c|}{ CSF NfL*Time } \\
\hline Neuroimaging measure(z-score) & $b(s e)$ & $\begin{array}{c}p- \\
\text { value }\end{array}$ & $b(s e)$ & $\begin{array}{c}p- \\
\text { value }\end{array}$ \\
\hline \multicolumn{5}{|l|}{ Cortical Thickness } \\
\hline Frontal & $-0.229(0.045)$ & $<0.001$ & $-0.016(0.075)$ & 0.836 \\
\hline Parietal & $-0.167(0.043)$ & $<0.001$ & $0.052(0.071)$ & 0.465 \\
\hline Occipital & $-0.077(0.042)$ & 0.070 & $0.145(0.068)$ & 0.034 \\
\hline Temporal & $-0.144(0.043)$ & $<0.001$ & $-0.042(0.067)$ & 0.537 \\
\hline$A D$ signature region & $-0.162(0.045)$ & $<0.001$ & $-0.075(0.072)$ & 0.302 \\
\hline \multicolumn{5}{|l|}{ White Matter Indices } \\
\hline Corpus Callosum FA & $-0.134(0.068)$ & 0.048 & $-0.283(0.096)$ & 0.003 \\
\hline Cingulum FA & $-0.140(0.062)$ & 0.025 & $-0.308(0.112)$ & 0.006 \\
\hline WMH Fraction & $0.061(0.064)$ & 0.340 & $0.085(0.080)$ & 0.288 \\
\hline
\end{tabular}

Multivariable models adjust for age, sex, and CSF A $\beta$ 
Table 3. Interactions between each CSF ' $N$ ' marker and CSF A $\beta$ for both cross-sectional and longitudinal neuroimaging

\section{outcomes}

\begin{tabular}{|c|c|c|c|c|c|c|c|c|c|c|c|c|c|c|}
\hline \multirow[b]{2}{*}{ Neuroimaging (z-score) } & \multicolumn{2}{|c|}{ CSF Total tau } & \multicolumn{2}{|c|}{ CSF At } & \multicolumn{2}{|l|}{ Time } & \multicolumn{2}{|c|}{ CSF Total tau *Time } & \multicolumn{2}{|c|}{ CSF Total tau*A+ } & \multicolumn{2}{|c|}{ CSF A+ *Time } & \multicolumn{2}{|c|}{ CSF Total Tau*A $+{ }^{*}$ Time } \\
\hline & $b(s e)$ & $p$-value & $b(s e)$ & $p$-value & $b(s e)$ & $p$-value & $b(s e)$ & $p$-value & $b(s e)$ & $p$-value & $b(s e)$ & $p$-value & $b(s e)$ & $p$-value \\
\hline \multicolumn{15}{|l|}{ Cortical Thickness } \\
\hline Frontal & $-0.028(0.074)$ & 0.704 & $0.029(0.085)$ & 0.738 & $-0.075(0.101)$ & 0.454 & $-0.041(0.127)$ & 0.746 & $-0.049(0.090)$ & 0.587 & $-0.429(0.151)$ & 0.004 & $-0.116(0.159)$ & 0.466 \\
\hline Parietal & $-0.018(0.070)$ & 0.791 & $0.073(0.080)$ & 0.362 & $-0.431(0.096)$ & $<0.001$ & $0.064(0.121)$ & 0.595 & $-0.072(0.084)$ & 0.395 & $-0.399(0.143)$ & 0.006 & $-0.188(0.151)$ & 0.214 \\
\hline Occipital & $0.117(0.068)$ & 0.085 & $0.093(0.078)$ & 0.231 & $-0.509(0.092)$ & $<0.001$ & $0.080(0.116)$ & 0.492 & $-0.166(0.082)$ & 0.043 & $-0.333(0.137)$ & 0.016 & $-0.135(0.145)$ & 0.353 \\
\hline Temporal & $0.194(0.069)$ & 0.005 & $0.025(0.079)$ & 0.753 & $-0.682(0.089)$ & $<0.001$ & $-0.055(0.112)$ & 0.626 & $-0.222(0.084)$ & 0.008 & $-0.467(0.134)$ & $<0.001$ & $-0.254(0.141)$ & 0.073 \\
\hline$A D$ signature region & $0.128(0.072)$ & 0.078 & $-0.018(0.083)$ & 0.832 & $-0.722(0.096)$ & $<0.001$ & $-0.147(0.121)$ & 0.222 & $-0.204(0.087)$ & 0.020 & $-0.398(0.143)$ & 0.006 & $-0.247(0.151)$ & 0.102 \\
\hline \multicolumn{15}{|l|}{ Diffusion Tensor Imaging } \\
\hline Corpus Callosum FA & $0.318(0.131)$ & 0.016 & $-0.031(0.130)$ & 0.814 & $0.121)$ & $<0.001$ & $0.037(0.168)$ & 0.826 & $-0.156(0.156)$ & 0.317 & $-0.070(0.184)$ & 0.701 & -0.29 & 0.160 \\
\hline Cingulate $C$ & $-0.096(0.122)$ & 0.432 & $-0.061(0.121)$ & 0.615 & $-1.010(0.141)$ & $<0.001$ & $0.175(0.196)$ & 0.371 & $-0.056(0.145)$ & 0.701 & $0.017(0.213)$ & 0.936 & $-0.478(0.242)$ & 0.049 \\
\hline \multirow[t]{2}{*}{ WMH Fraction } & $-0.269(0.127)$ & 0.035 & $0.122(0.126)$ & 0.337 & $0.473(0.102)$ & $<0.001$ & $0.065(0.142)$ & 0.647 & $0.330(0.149)$ & 0.029 & $0.266(0.144)$ & 0.067 & $0.045(0.167)$ & 0.789 \\
\hline & \multicolumn{2}{|c|}{$\underline{\mathrm{CSF} \mathrm{Ng}}$} & \multicolumn{2}{|l|}{$\underline{\operatorname{CSF} A_{+}}$} & \multicolumn{2}{|l|}{$\underline{\text { Time }}$} & \multicolumn{2}{|c|}{$\underline{\text { CSF Ng*Time }}$} & \multicolumn{2}{|c|}{$\underline{\operatorname{CSF~Ng}{ }^{*} \mathrm{~A}+}$} & \multicolumn{2}{|c|}{$\underline{\text { CSF A+ *Time }}$} & \multicolumn{2}{|c|}{$\underline{\mathrm{CSF} \mathrm{Ng}{ }^{*} \mathrm{~A}+{ }^{*} \mathrm{Time}}$} \\
\hline Neuroimaging (z-score) & $b(s e)$ & $p$-value & $b(s e)$ & $p$-value & (se) & ue & $b(s e)$ & $p$-value & $b(s e)$ & $p$-value & $b(s e)$ & $p$-value & $b(s e)$ & $p$-value \\
\hline \multicolumn{15}{|l|}{ Cortical Thickness } \\
\hline Fron & $-0.025(0.068)$ & 0.708 & $014(0.085)$ & 0.708 & $4(0.100)$ & 298 & $0.077(0.117)$ & 0.512 & $-0.100(0.089)$ & 0.260 & $-0.419(0.150)$ & 0.006 & $-0.206(0.158)$ & 0.194 \\
\hline Pari & $-0.031(0.064)$ & 0.626 & $9(0.080)$ & 0.458 & $45(0.095)$ & $<0.001$ & $0.120(0.111)$ & 0.280 & $-0.069(0.083)$ & 0.408 & $-0.401(0.143)$ & 0.005 & $-0.261(0.150)$ & 0.083 \\
\hline Occipital & $0.063(0.062)$ & 0.313 & $0.076(0.078)$ & 0.331 & $-0.490(0.092)$ & $<0.001$ & $0.005(0.107)$ & 0.962 & $-0.116(0.081)$ & 0.154 & $-0.363(0.137)$ & 0.008 & $-0.140(0.144)$ & 0.334 \\
\hline Temporal & $0.128(0.064)$ & 0.045 & $0.006(0.080)$ & 0.945 & $-0.724(0.089)$ & $<0.001$ & $0.112(0.104)$ & 0.283 & $-0.177(0.083)$ & 0.033 & $-0.467(0.133)$ & $<0.001$ & $-0.464(0.140)$ & $<0.001$ \\
\hline$A D$ signature region & $0.078(0.066)$ & 0.242 & $-0.036(0.083)$ & 0.667 & $-0.772(0.096)$ & $<0.001$ & $0.052(0.112)$ & 0.641 & $-0.158(0.086)$ & 0.068 & $-0.400(0.143)$ & 0.005 & $-0.471(0.151)$ & 0.002 \\
\hline \multicolumn{15}{|l|}{ Diffusion Tensor Imaging } \\
\hline Corpus Callosum FA & $0.216(0.114)$ & 0.060 & $0.002(0.130)$ & 0.990 & $.260(0.122)$ & $<0.001$ & $-0.027(0.157)$ & 0.862 & $-0.007(0.144)$ & 0.960 & $-0.087(0.184)$ & 0.637 & $-0.323(0.207)$ & 0.120 \\
\hline Cingulate Gyrus FA & $-0.046(0.107)$ & 0.671 & $-0.028(0.121)$ & 0.820 & $-1.029(0.182)$ & 0.189 & $0.240(0.182)$ & 0.189 & $-0.028(0.135)$ & 0.837 & $0.022(0.213)$ & 0.918 & $-0.662(0.240)$ & 0.006 \\
\hline \multirow[t]{2}{*}{ WMH Fraction } & $-0.244(0.112)$ & 0.031 & $0.018(0.124)$ & 0.882 & $0.474(0.102)$ & $<0.001$ & $-0.092(0.135)$ & 0.498 & $0.188(0.137)$ & 0.173 & $0.255(0.145)$ & 0.079 & $0.161(0.165)$ & 0.330 \\
\hline & \multicolumn{2}{|l|}{$\underline{\text { CSF NfL }}$} & \multicolumn{2}{|c|}{$\underline{\mathrm{CSF} A+}$} & \multicolumn{2}{|l|}{$\underline{\text { Time }}$} & $\underline{\text { CSF NfL }{ }^{*} T}$ & Time & $\underline{\text { CSF NfL }}$ & & $\underline{\operatorname{CSF} A+}$ & ime & $\underline{\text { CSF NfL* }}$ & ${ }^{*}$ Time \\
\hline Neuroimaging (z-score) & $b(s e)$ & $p$-value & $b(s e)$ & $p$-value & $b(s e)$ & $p$-value & $b(s e)$ & $p$-value & $b(s e)$ & $p$-value & $b(s e)$ & $p$-value & $b(s e)$ & $p$-value \\
\hline Cortical Thickness & & & & & & & & & & & & & & \\
\hline $\mathrm{F}$ & $-0.200(0.059)$ & $<0.001$ & $13(0.082)$ & 0.873 & $97(0.098)$ & 1 & $0.053(0.097)$ & 0.583 & $-0.060(($ & 0.484 & $-0.414(0.148)$ & 0.005 & -0.214( & 0.162 \\
\hline$P$ & $-0.162(0.056)$ & 0.004 & $0.060(0.078)$ & 0.440 & $-0.448(0.092)$ & $<0.001$ & $0.167(0.092)$ & $<0.001$ & $-0.025(0.081)$ & 0.759 & $-0.385(0.141)$ & 0.006 & $-0.328(0.145)$ & 0.024 \\
\hline Occi & $-0.081(0.055)$ & 0.141 & $0.059(0.077)$ & 0.445 & $-0.531(0.089)$ & $<0.001$ & $0.222(0.088)$ & 0.012 & $-0.029(0.079)$ & 0.714 & $-0.317(0.135)$ & 0.019 & $-0.223(0.139)$ & 0.108 \\
\hline Temporal & $-0.118(0.056)$ & 0.036 & $-0.031(0.078)$ & 0.688 & $-0.691(0.087)$ & $<0.001$ & $-0.028(0.087)$ & 0.751 & $-0.042(0.081)$ & 0.606 & $-0.476(0.133)$ & $<0.001$ & $-0.080(0.137)$ & 0.560 \\
\hline $\begin{array}{l}\text { AD signature region } \\
\text { Diffusion Tensor Imaging }\end{array}$ & -0.134 & 0.022 & & 0.475 & $-0.747(0.094)$ & & $-0.067(0.093)$ & 0.472 & .084) & 0.646 & $99(0.143)$ & 0.006 & $054(0.147)$ & 0.712 \\
\hline Corpus Callosum FA & $-0.162(0.092)$ & 0.078 & $-0.097(0.128)$ & 0.451 & $-1.286(0.120)$ & $<0.001$ & $-0.216(0.120)$ & 0.072 & $0.133(0.131)$ & 0.310 & $-0.086(0.183)$ & 0.638 & $-0.183(.200)$ & 0.360 \\
\hline Cingulate Gyrus FA & $-0.050(0.085)$ & 0.553 & $-0.031(0.118)$ & 0.796 & $-1.027(0.141)$ & $<0.001$ & $-0.304(0.140)$ & 0.031 & $-0.112(0.121)$ & 0.355 & $-0.002(0.214)$ & 0.994 & $-0.020(0.234)$ & 0.933 \\
\hline WMH Fraction & $-0.026(0.093)$ & 0.780 & $0.135(0.127)$ & 0.288 & $0.489(0.110)$ & $<0.001$ & $0.054(0.111)$ & 0.630 & $0.147(0.127)$ & 0.248 & $0.263(0.154)$ & 0.088 & $0.057(0.160)$ & 0.723 \\
\hline
\end{tabular}

Models adjust for age and sex. 
Figure 1. Longitudinal Associations Between CSF T-tau and NfL with MRI Cortical Thickness and White Matter Indices.

Figure 2. Interactions Between CSF Ng and Amyloid for Change in MRI Cortical Thickness and White Matter Indices.

Supplementary Table 1. Multivariable Associations between Each CSF 'N' Marker and both cross-sectional and Longitudinal Neuroimaging Outcomes Among Participants with all Neuroimaging Measures

Supplementary Table 2. Interactions between each CSF ' $N$ ' marker and CSF A $\beta$ for both cross-sectional and longitudinal neuroimaging outcomes among those with all imaging modalities 